\title{
Ab initio calculations of oscillator strengths and Landé factors for Nd III
}

\author{
D.J. Bord ${ }^{1,2}$ \\ 1 Department of Natural Sciences, University of Michigan-Dearborn, 4901 Evergreen Rd., Dearborn, MI 48128, U.S.A. \\ 2 Department of Astronomy, University of Michigan-Ann Arbor, 953 Dennison Building, Ann Arbor, MI 48109-1090, U.S.A.
}

Received March 3; accepted April 14, 2000

\begin{abstract}
Theoretical calculations of oscillator strengths and Landé $g$-factors made with the Cowan code are presented for the rare-earth ion NdIII. The emphasis is on transitions in the optical region of the spectrum. Comparisons of previous ab initio calculations of this type for other lanthanides (e.g., LaII, CeIII, and LuII) with published experimental and theoretical data suggest that the accuracy of the $\log (g f)$ values for individual transitions should be about \pm 0.15 dex in the absence of significant core polarization effects; the $g$-factors should be good to better than $5 \%$, except in a few cases where term mixing is important. Applications of the data are made in the calculation of neodymium abundances in the atmospheres of the chemically peculiar stars HD 101065 ("Przybylski's star") and HD 122970.
\end{abstract}

Key words: atomic data — line: formation — stars: chemically peculiar — stars: abundances

\section{Introduction}

The need for reliable atomic data in the study of astrophysical problems is well-known (see, e.g., the summary by Baschek 1995). In spectrum synthesis work, particularly as applied to chemically peculiar (CP) stars, accurate knowledge of transition probabilities and oscillator strengths for heavy and rare-earth elements (REE) is essential to establishing reliable abundances for these species. Available data of this type for the first and second spectra of the lanthanide REE are spotty (see Fig. 2 in Wiese et al. 1995), but very little is known about the $g f$-values for the third spectra of these elements. Since features associated with doubly ionized lanthanide REE are increasingly being identified in high-dispersion, high signal-to-noise spectra of CP stars (see Leckrone et al. 1998; Cowley et al. 1998, 2000; Ryabchikova et al. 1999), it is becoming critically important for our understanding of the atmospheric structure and abundance patterns in these stars to have more information about the atomic parameters characterizing these species.

In an attempt to meet this need, our group has carried out ab initio calculations of oscillator strengths for La II (Bord et al. 1996), Ce III (Bord et al. 1997), Nd III (Cowley \& Bord 1998), and Lu II (Bord et al. 1998) using the Cowan code (Cowan 1981, 1985), and we have applied these results in abundance analyses of several CP stars and the Sun. In this paper, a more expansive list of $g f$-values for predicted features of Nd III than was given in our earlier work on $\gamma$ Equulei and HR 6870 is presented. Landé $g$-factors are also tabulated so that magnetic field effects may be modeled in cases where they are expected to be important. In what follows, the methodology employed in carrying out these calculations is briefly discussed (Sect. 2) before the results are introduced in Sect. 3. Section 4 contains some commentary on the expected accuracy of the results based on comparisons with existing data. Section 5 concludes with some applications of the data that are currently being made by our group.

\section{Methodology}

The general methodology employed in making the calculations reported herein continues to be based on the Cowan code (Cowan 1981, 1985), and has been described in previous papers. Although some of the details of the Nd III calculations have appeared before (Cowley \& Bord 1998), we review here the principal features of the analysis.

Single-particle radial wavefunctions were determined using a Hartree-plus-statistical-exchange interaction approximation for the following even and odd parity configurations, respectively: $\left(4 \mathrm{f}^{4}+4 \mathrm{f}^{2} 5 \mathrm{~d}^{2}+4 \mathrm{f}^{2} 5 \mathrm{~d} 6 \mathrm{~s}+4 \mathrm{f}^{3} 6 \mathrm{p}\right)$, and $\left(4 \mathrm{f}^{3} 5 \mathrm{~d}+4 \mathrm{f}^{3} 6 \mathrm{~s}\right)$. An additional odd configuration, $4 \mathrm{f}^{2} 5 \mathrm{~d} 6 \mathrm{p}$, was found to lie too high in energy to be of importance. Experimental energy levels exist for only two of these configurations $\left(4 \mathrm{f}^{4}\right.$ and $\left.4 \mathrm{f}^{3} 5 \mathrm{~d}\right)$, and neither is complete (Martin et al. 1978, hereafter MZH). The selected 
configurations comprise all of Goldschmidt's (1978) system $A$ and $B$ configurations (with the exception of $4 \mathrm{f}^{2} 6 \mathrm{~s}^{2}$ ) and are well isolated from adjoining, higher ones which should minimize perturbations known to reduce the accuracy of the calculations. As in the past, relativistic and electron correlation corrections have been included in the calculations, and the eigenvectors were constructed using both LS- and jj-coupling basis sets. LS coupling is generally appropriate for the levels included in this study, except for those arising from the $4 \mathrm{f}^{3} 6 \mathrm{~s}$ and $4 \mathrm{f}^{3} 6 \mathrm{p}$ configurations, where a $J_{1} j$-coupling scheme is preferred.

$\mathrm{Ab}$ initio values for the single-configuration center-ofgravity energies and the various radial and configuration interaction integrals were computed with uniform scaling applied to all spin-orbit and Slater parameters. Reducing the theoretical values of $\mathrm{F}^{k}$ and $\mathrm{G}^{k}$ in this manner, for example, roughly accounts for the effects of introducing additional two-body electrostatic operators for legal values of $k$ (Cowan 1981, p. 478); no attempt to include effects due to "illegal- $k$ " effective operators has been made.

The radial integrals were then optimized to fit the known energy levels using the method of least squares. Since experimental Landé factors are not available for Nd III, additional improvements to the eigenvectors, particularly those not well described by LS-coupling, by including fits to the $g$-factors (Cowan 1981, p. 473) could not be performed.

For Nd III, we computed a total of 1488 levels, of which only 28 ( 5 even and 23 odd) were fitted. One odd level at $24003 \mathrm{~cm}^{-1}$ with $J=7$ and no term designation in MZH was omitted from the fit. The adopted structure parameters yielded a mean deviation in energy of less than $1 \mathrm{~cm}^{-1}\left(0.02 \%\right.$ over $\left.\approx 5100 \mathrm{~cm}^{-1}\right)$ for the even levels and only $54 \mathrm{~cm}^{-1}$ (about $0.2 \%$ over $\approx 33000 \mathrm{~cm}^{-1}$ ) for the odd levels.

Term designations and leading percentages for the known levels analyzed typically agreed with those given in MZH to within $10 \%$ in cases where the leading percentages were $\geq 50 \%$. In instances where term mixing is severe and no assignment is given by MZH, the calculations usually identified the level with one of the two principal terms given in the NIST compilation. Exceptions were found for three terms: the ${ }^{5} \mathrm{I}_{5}^{\circ}$ at $20389 \mathrm{~cm}^{-1}$ and ${ }^{5} \mathrm{H}_{4}^{\circ}$ at $28745 \mathrm{~cm}^{-1}$ showed leading percentages $\approx 20 \%$ less than those given in MZH at $56 \%$ and $63 \%$, respectively; and the ${ }^{5} \mathrm{H}_{6}^{\circ}$ at $31395 \mathrm{~cm}^{-1}$ was computed to be substantially purer than indicated in MZH with a leading percentage of $72 \%$ as compared to $46 \%$.

\section{Results}

Table 1 lists the known energy levels for Nd III from MZH and the Landé $g$-factors calculated using the Cowan code.

$\log (g f)$ values were computed for all electric dipole transitions with wavelengths in the range $2000-7000 \AA$.
Table 1. Landé $g$-factors for $\mathrm{Nd} \mathrm{III}^{a}$

\begin{tabular}{|c|c|c|c|c|}
\hline Configuration & Term & $J$ & $\begin{array}{l}\text { Energy } \\
\left(\mathrm{cm}^{-1}\right)\end{array}$ & $g$-factor \\
\hline \multirow{5}{*}{$4 \mathrm{f}^{4}$} & ${ }^{5} \mathrm{I}$ & 4 & 0.0 & 0.605 \\
\hline & & 5 & 1137.8 & 0.902 \\
\hline & & 6 & 2387.6 & 1.071 \\
\hline & & 7 & 3714.9 & 1.176 \\
\hline & & 8 & 5093.3 & 1.246 \\
\hline \multirow[t]{5}{*}{$4 f^{3}\left({ }^{4} I^{\circ}\right) 5 d$} & ${ }^{5} \mathrm{~K}^{\circ}$ & 5 & 15262.2 & 0.683 \\
\hline & & 6 & 16938.1 & 0.909 \\
\hline & & 7 & 18656.3 & 1.052 \\
\hline & & 8 & 20410.9 & 1.149 \\
\hline & & 9 & 22197.0 & 1.217 \\
\hline \multirow[t]{5}{*}{$4 f^{3}\left({ }^{4} I^{\circ}\right) 5 d$} & ${ }^{5} \mathrm{I}^{\circ}$ & 4 & 18883.7 & 0.621 \\
\hline & & 5 & 20388.9 & 0.930 \\
\hline & & 6 & 22047.8 & 1.079 \\
\hline & & 7 & 22702.9 & 1.153 \\
\hline & & 8 & 24686.4 & 1.232 \\
\hline \multirow[t]{4}{*}{$4 f^{3}\left({ }^{4} I^{\circ}\right) 5 d$} & ${ }^{5} \mathrm{H}^{\circ}$ & 3 & 19211.0 & 0.538 \\
\hline & & 4 & 20144.3 & 0.873 \\
\hline & & 5 & 21886.8 & 1.026 \\
\hline & & 6 & 23819.3 & 1.073 \\
\hline $4 f^{3}\left({ }^{4} I^{\circ}\right) 5 d$ & & 7 & 24003.2 & 1.184: \\
\hline $4 f^{3}\left({ }^{4} I^{\circ}\right) 5 d$ & & 6 & 26503.2 & 1.183: \\
\hline $4 f^{3}\left({ }^{4} I^{\circ}\right) 5 d$ & ${ }^{3} \mathrm{~K}^{\circ}$ & 8 & 27391.4 & 1.137 \\
\hline $4 \mathrm{f}^{3}\left({ }^{4} \mathrm{~F}^{\circ}\right) 5 \mathrm{~d}$ & & 3 & 27569.8 & 1.146: \\
\hline \multirow[t]{5}{*}{$4 \mathrm{f}^{3}\left({ }^{4} \mathrm{~F}^{\circ}\right) 5 \mathrm{~d}$} & ${ }^{5} \mathrm{H}^{\circ}$ & 3 & 27788.2 & 0.807 \\
\hline & & 4 & 28745.3 & 1.074 \\
\hline & & 5 & 30232.3 & 1.126 \\
\hline & & 6 & 31394.6 & 1.208 \\
\hline & & 7 & 32832.6 & 1.158 \\
\hline $4 \mathrm{f}^{3}\left({ }^{4} \mathrm{~F}^{\circ}\right) 5 \mathrm{~d}$ & & 5 & 29297.3 & 1.126: \\
\hline
\end{tabular}

${ }^{a}$ Energies and term designations are from Martin et al. (1978); $g$-factors followed by a colon are uncertain due to term mixing.

A total of 9469 lines and associated $g f$-values exist in our data files. To present a complete list of the calculations is beyond the scope of this note. Table 2 shows the calculated $g f$-values, as well as the cancellation factors $(\mathrm{CF}$, see Sect. 4), for the 54 predicted lines arising from the known energy levels after employing the usual selection rules; the $g f$-values have been scaled appropriately for the differences between the calculated wavelengths and the predicted ones.

Fewer than half of these lines appear in unpublished lists of the strongest laboratory lines of Nd III provided by Crosswhite (1976), but many of these have been identified in RE-rich stars like HR 465 (Bidelman et al. 1995) and HD 101065 (Cowley et al. 1998, 2000). Hundreds of 
additional strong lines exist in the Crosswhite data, but it is difficult, if not impossible, given the uncertainties in the calculated energy levels, to unambiguously classify them using our data. The full set of computed wavelengths, energy levels, and $\log (g f)$ values will be released to the Vienna Atomic Line Database (VALD, Piskunov et al. 1995; Kupka et al. 1999) for the purpose of computing opacities for heavily line-blanketed stellar model atmospheres where especially high precision in the wavelengths is not required and where the $f$-sum rule for the oscillator strengths mitigates somewhat the uncertainties in the individual values.

No corrections to the $g f$-values given in Table 2 for core polarization effects have been made due to the fact that our current version of the multi-configuration Hartree-Fock-based code for doing so (see Vaeck et al. 1992) will not handle configurations containing more than two $f$-electrons. We do not expect this to be a significant limitation on the accuracy of the data in Table 2 insofar as it contains no transitions into or out of any levels assigned to the $4 \mathrm{f}^{3} 6 \mathrm{p}$ configuration where such effects are most pronounced. A modified program to permit core polarization corrections to be made in configurations with greater than two $f$-electrons has been kindly made available to us by G. Gaigalas (1998) and awaits implimentation.

Based on the calculations, a re-evaluation of the ionization energy for $\mathrm{Nd}$ III has been carried out following the approach of Sugar \& Reader (1973). Using the computed energy for the lowest level of the $4 \mathrm{f}^{3} 6 \mathrm{~s}$ configuration, $30776 \mathrm{~cm}^{-1}$, and taking $\delta=455 \mathrm{~cm}^{-1}$ (as found from the formulae of Judd (1962) and the calculated Slater parameter $\left.\mathrm{G}^{3}\left(4 \mathrm{f}^{3} 6 \mathrm{~s}\right)=2125 \mathrm{~cm}^{-1}\right)$, we find the ionization energy to be $179031 \pm 823 \mathrm{~cm}^{-1}$. This result is only $0.2 \%$ higher than the published value, but it reduces the uncertainty by nearly a factor of three over the previous estimate. In this determination, we have applied the Sugar \& Reader term value for the center of gravity of the $4 \mathrm{f}^{3} 6 \mathrm{~s}$ configuration derived from the Rydberg-Ritz formula: $T=147800 \mathrm{~cm}^{-1}$. The uncertainty in the ionization energy is the square root of the sum of the squares of the contributing uncertainites. We have adopted the value given in Sugar \& Reader for $\Delta T$ and assumed the error in the energy for the $4 \mathrm{f}^{3} 6 \mathrm{~s}^{5} \mathrm{I}_{4}^{\circ}$ term to be three times the mean deviation in the fitted energies for the odd parity levels.

\section{Reliability of the data}

\section{1. gf-values}

One of the major purposes of performing these calculations is to apply them in the determination of REE abundances in CP stars. Given the high quality of modern spectroscopic observations and the increasing sophistication of stellar model atmosphere codes, the limiting
Table 2. $g f$-values for predicted lines in Nd III

\begin{tabular}{|c|c|c|c|c|}
\hline $\begin{array}{c}\lambda_{\text {pred }} \\
(\AA)\end{array}$ & $\begin{array}{r}\lambda_{\exp }{ }^{a} \\
\quad(\AA)\end{array}$ & Transition & $\log (g f)$ & $\mid C F$ \\
\hline 3283.67 & .65 & $2387.6_{6}-32832.6_{7}^{\circ}$ & -3.23 & 0.12 \\
\hline 3304.09 & & $1137.8_{5}-31394.6_{6}^{\circ}$ & -3.24 & 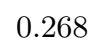 \\
\hline 3306.77 & & $0_{4}-30232.3_{5}^{\circ}$ & -3.28 & .107 \\
\hline 3400.70 & & $0_{4}-29397.3_{5}^{\circ}$ & -4.98 & .015 \\
\hline 3433.35 & .33 & $3714.9_{7}-32832.6_{7}^{\circ}$ & -1.61 & 315 \\
\hline 3436.09 & & $1137.8_{5}-30232.3_{5}^{\circ}$ & -1.60 & 0.568 \\
\hline 3446.46 & & $2387.6_{6}-31394.6_{6}^{\circ}$ & -1.57 & 0.633 \\
\hline 3477.83 & .83 & $0_{4}-28745.3_{4}^{\circ}$ & -1.87 & 0641 \\
\hline 3537.62 & .61 & $1137.8_{5}-29397.3_{5}^{\circ}$ & -3.29 & .107 \\
\hline 3590.32 & .33 & $2387.6_{6}-30232.3_{5}^{\circ}$ & -0.76 & 0.69 \\
\hline 3597.62 & .62 & $0_{4}-27788.2_{3}^{\circ}$ & -0.81 & 0.839 \\
\hline 3603.97 & .98 & $5093.3_{8}-32832.6_{7}^{\circ}$ & -0.63 & 0.697 \\
\hline 3611.73 & .72 & $3714.9_{7}-31394.6_{6}^{\circ}$ & -0.49 & \\
\hline 3621.17 & .17 & $1137.8_{5}-28745.3_{4}^{\circ}$ & -0.74 & 0.830 \\
\hline 3626.12 & .12 & $0_{4}-27569.8_{3}^{\circ}$ & -1.24 & 0.730 \\
\hline 3701.32 & .29 & $2387.6_{6}-29397.3_{5}^{\circ}$ & -2.29 & 0.32 \\
\hline 3941.26 & .26 & $1137.8_{5}-265$ & -3.81 & 0.035 \\
\hline 4145.52 & .50 & $2387.6_{6}-26$ & -2.78 & 0.070 \\
\hline 4222.41 & & $3714.9_{7}-27391.4_{8}^{\circ}$ & -2.81 & 0.1 \\
\hline 4386.98 & & $3714.9_{7}-26503.2_{6}^{\circ}$ & -2.41 & 0.093 \\
\hline 4407.64 & & $1137.8_{5}-23819.3_{6}^{\circ}$ & -3.72 & 0.014 \\
\hline 4483.43 & .44 & $5093.3_{8}-27391.4_{8}^{\circ}$ & -1.43 & 0.219 \\
\hline 4567.68 & & $0_{4}-21886.8_{5}^{\circ}$ & -3.43 & 0.026 \\
\hline 4624.99 & .96 & $2387.6_{6}-24003.2_{7}^{\circ}$ & -2.01 & 0.387 \\
\hline 4664.68 & & $2387.6_{6}-23819.3_{6}^{\circ}$ & -3.22 & 0.013 \\
\hline 4767.04 & & $3714.9_{7}-24686.4_{8}^{\circ}$ & -1.72 & 0.623 \\
\hline 4781.06 & & $1137.8_{5}-22$ & -1.71 & 0.455 \\
\hline 4818.16 & & $1137.8_{5}-21886.8_{5}^{\circ}$ & -3.70 & 0.003 \\
\hline 4903.26 & & $0_{4}-20388.9_{5}^{\circ}$ & -1.86 & 0.366 \\
\hline 4921.02 & & $2387.6_{6}-22702.9_{7}^{\circ}$ & -1.77 & 0.560 \\
\hline 4927.57 & $.48 ?$ & $3714.9_{7}-24$ & -0.86 & 0.502 \\
\hline 4962.80 & & $0_{4}-20144.3_{4}^{\circ}$ & -6.08 & 0.000 \\
\hline 4972.65 & & $3714.9_{7}-23819.3_{6}^{\circ}$ & -1.27 & 0.225 \\
\hline 5085.00 & & $2387.6_{6}-22047.8_{6}^{\circ}$ & -0.68 & 0.583 \\
\hline 5102.42 & .43 & $5093.3_{8}-2$ & -0.40 & 0.699 \\
\hline 5126.99 & 7.00 & $2387.6_{6}-21$ & -1.09 & 0.306 \\
\hline 193.06 & .06 & $1137.8_{5}-20388.9_{5}^{\circ}$ & -0.77 & 0.597 \\
\hline 5203.90 & .91 & $0_{4}-19211.0_{3}^{\circ}$ & -1.19 & 0.32 \\
\hline 5259.89 & & $1137.8_{5}-20$ & -1.15 & 0.350 \\
\hline 5265.02 & $4.96 ?$ & $3714.9_{7}-22$ & -0.67 & 0.572 \\
\hline 5286.76 & & $5093.3_{8}-24003.2_{7}^{\circ}$ & -1.60 & 0.087 \\
\hline 5294.10 & .11 & $0_{4}-18883.7_{4}^{\circ}$ & -0.67 & 0.709 \\
\hline 5453.16 & & $3714.9_{7}-22047.8_{6}^{\circ}$ & -7.52 & 0.000 \\
\hline 5553.61 & & $2387.6_{6}-20388.9_{5}^{\circ}$ & -3.74 & 0.002 \\
\hline 5633.54 & & $1137.8_{5}-18883.7_{4}^{\circ}$ & -2.01 & 0.340 \\
\hline 5677.15 & & $5093.3_{8}-22702.9_{7}^{\circ}$ & -1.42 & 0.390 \\
\hline 5845.07 & $.00 ?$ & $5093.3_{8}-22197.0_{9}^{\circ}$ & -1.16 & 0.581 \\
\hline 5987.80 & & $3714.9_{7}-20410.9_{8}^{\circ}$ & -1.25 & 0.546 \\
\hline 6145.07 & $.02 ?$ & $2387.6_{6}-18656.3_{7}^{\circ}$ & -1.33 & 0.540 \\
\hline 6327.24 & .22 & $1137.8_{5}-16938.1_{6}^{\circ}$ & -1.40 & 0.551 \\
\hline 6526.63 & & $5093.3_{8}-20410.9_{8}^{\circ}$ & -2.34 & 0.552 \\
\hline 6550.33 & $.21 ?$ & $0_{4}-15262.2_{5}^{\circ}$ & -1.47 & 0.557 \\
\hline 6690.97 & & $3714.9_{7}-18656.3_{7}^{\circ}$ & -2.29 & 0.51 \\
\hline 6870.72 & & $2387.6_{6}-16938.1_{6}^{\circ}$ & -2.43 & 0.47 \\
\hline
\end{tabular}

${ }^{a}$ Based on data provided by Crosswhite (1976); entries with questions are possible identifications, although the wavelength differences are large. 
factor in producing accurate abundances for many heavy and REE is the uncertainty in their atomic parameters, particularly the transition probabilities and/or $g f$-values. On the logarithmic scale typically used to report stellar abundances, the errors in element abundances scale directly with those in the $\log (g f)$ values. Consequently, it is important to assess the reliability of the $g f$-values presented here.

Based on previous experience, as well as on comparisons with data released since the appearance of some of our earlier work, we expect that, for lines with cancellation factors substantially greater than 0.1 in absolute value (cf. Cowan 1981, Eq. 14.107), and discounting core polarization effects, the oscillator strengths should be accurate to \pm 0.15 dex. For transition arrays in which core polarization is important, most notably those involving $p$-electrons, we have found that our calculated radiative lifetimes can be up to a factor of 2 too small when compared with experiment (cf. Bord et al. 1997); in these cases, the calculated $\log (g f)$ values are likely to be systematically too large by some 0.25 dex or more on average. As noted above, for the data reported here which do not involve configurations containing $p$-electrons, we do not anticipate that core polarization effects will contribute significantly to producing uncertainties in the $g f$-values beyond the quoted limits.

Since no experimental or theoretical $g f$-values exist for this ion to the author's knowledge, indirect evaluations of our computations must be made. In support of our assessment of the data, the following comparisions may be relevant. For La II, our recommended formula for computing $g f$-values, based on a calibration of NBS Monograph 145 (Meggers et al. 1975) intensities using Cowan-code oscillator strengths (Bord et al. 1996), produces agreement with values computed by Gratton \& Sneden (1994) from experimental lifetime data to within 0.02 dex on average, with a scatter of only \pm 0.08 dex. Similarly, for Lu II, comparison of results found using our $\log (g f)$ formula, again derived from Cowan-code calibrated Monograph 145 intensities (Bord et al. 1998), with recently published experimental $g f$-values (Quinet et al. 1999) for 10 lines yields a mean and standard deviation of only $-0.026 \pm$ 0.177 dex. Finally, an examination of the $g f$-values recently published by Wyart \& Palmeri (1998) in their comprehensive study of Ce III finds remarkably good agreement with those appearing in Bord et al. (1997), especially given that the former study incorporated ten new and/or revised energy levels and included more than twice the number of even and odd configurations used by us; neither investigation, however, includes corrections for core polarization effects. In particular, for the 30 lines held in common in the published lists with cancellation factors large enough to make the theoretical transition probabilities reliable, the mean difference and standard error in the $\log (g f)$ 's between the two investigations (taken in the sense $\left.\log \left(g f_{\mathrm{BCM}}\right)-\log \left(g f_{\mathrm{WP}}\right)\right)$ is only $-0.014 \pm 0.133$.

\section{2. g-factors}

To the author's knowledge, no experimental measures of the $g$-factors for NdIII have been made. To assess the expected accuracy of the values reported in this paper, appeal is again made to comparisons involving our prior work with other published studies. For example, for LaII where our term assignments and leading percentages are in excellent agreement with those given in $\mathrm{MZH}$, we find similarly good agreement between our theoretical $g$-factors and the experimental ones compiled by MZH from Harrison et al. (1945); for 96 measures spanning both even and odd parity states, the average percent difference between the two sets of data is under $2 \%$, while the mean and standard deviation of the difference $\Delta g \equiv\left|g_{\text {meas }}-g_{\text {theory }}\right|=0.015 \pm 0.018$. For reference, the internal agreement between independent measurements of the same $g$-factor is \pm 0.004 .

Similarly, in CeIII, for 34 levels with measured $g$-factors where the Cowan code term assignments agree with those presented in $\mathrm{MZH}$, the average difference between the measured values and those computed is $3.25 \%$. In six other cases where term mixing leads to disagreements between the Cowan-code designations and those reported by $\mathrm{MZH}$, the average deviations never exceeded $25 \%$. It may be worth noting in connection with this ion that the six measured $g$-factors considered uncertain (and marked with colons [:]) by MZH due to incomplete or unresolved Zeeman patterns are included in the first group of 34 levels mentioned above, and all agree with our calculated values to within the estimated errors of measurement, viz., \pm 0.02 .

Finally, for LuII, eliminating dubious measures and those possibly affected by hfs and/or unaccounted for term interactions (see discussion in MZH, p. 404), the absolute difference, $\Delta g$, taken in the same sense as above, between the NIST-compiled values and our calculations is $0.034 \pm 0.024$, or about $3 \%$ on average. This may be compared with the stated uncertainties in the measurements of $\pm 0.02-0.03$. In the light of these results, we can expect that the $g$-factors for $\mathrm{Nd}$ III reported here should be good to better than $5 \%$ overall, and that in cases of significant term mixing where the theoretical designations are uncertain, errors of under $25 \%$ may be anticipated.

\section{Applications and conclusion}

With judicious application, the data presented in this paper may be used to determine for the first time the abundances from Nd III in CP stars. This is important in corroborating the abundances of this element derived from $\mathrm{Nd}^{+}$, typically the dominant species in the atmospheres of cooler CP stars. Such calculations may also provide evidence of unusual atmospheric conditions related to element stratification and/or non-LTE effects in cases 
of substantial disagreements between abundances derived from different stages of ionization (Proffitt et al. 1999).

Nd III has been observed in the spectrum of several CP stars having strong lines of Nd II, for example HR 465, HD 51418, and HD 200311 (Cowley 1976; Aikman et al. 1979); NdII and Nd III have also been found in the Si star HD 192913 (Cowley \& Crosswhite 1978; Ryabchikova et al. 1990). We have previously reported on NdIII in HR 6870 and $\gamma$ Equ (Cowley \& Bord 1998), and recent studies by our group have also revealed the strong presence of both Nd II and Nd III in the spectrum of HD 101065 (Cowley et al. 2000), a rapidly oscillating Ap (roAp) star and arguably one of the most chemically bizarre objects in the Galaxy.

We have applied the oscillator strength data presented herein in the analysis of this star using the Michigan spectrum synthesis programs (Cowley 1996). Adopting a model stellar atmosphere with $T_{\text {eff }}=6600 \mathrm{~K}$ and $\log (g)$ $=3.5$ which provided acceptable fits to the Balmer line profiles in high resolution $(R=\lambda / \Delta \lambda=80000)$, high signal-to-noise $(S / N \geq 200)$ echelle spectra, we find a logarithmic abundance of Nd III of $5.2 \pm 0.4$ on the usual scale where $\log (H)=12.0$; this result is based on 5 lines longward of $5000 \AA$ that were free of significant blends and had wavelengths in reasonable agreement with predicted values $(\Delta \lambda \leq 0.04 \AA)$. This value is about 5000 times the solar abundance of $\mathrm{Nd}$, but it comports well with the abundance of this element derived from our studies of the $\mathrm{Nd}$ II spectrum in this star, viz. $4.9 \pm 0.2$ dex based on 14 unblended lines with wavelength differences with respect to the laboratory values of $\leq 0.02 \AA$. These calculations assumed a microturbulent velocity $\xi_{\mathrm{t}}=1.0 \mathrm{~km} \mathrm{~s}^{-1}$. These results differ from those published by Cowley \& Mathys (1998) in part due to differences in the model stellar atmosphere adopted, but also because of the use of a corrupted data file in the earlier analysis (Cowley 1999); for additional details, the reader is referred to Cowley et al. (2000).

HD 101065 possesses a weak surface magnetic field of $\approx 2500$ G (Wolff \& Hagen 1976; Cowley \& Mathys 1998) which has not been explicitly taken into account in our analysis; attempts to introduce magnetic intensification effects by raising the the microturbulence to $2.0 \mathrm{~km} \mathrm{~s}^{-1}$ reduce the abundance of $\mathrm{Nd}$ in HD 101065 by about $0.7-$ 0.8 dex.

Ryabchikova et al. (1999a) have also identified Nd II and Nd III in the spectra of several other roAp stars; an analysis of one such object, HD 122970 (Ryabchikova et al. 2000), using our $g$-factors and $g f$-values, has demonstrated that this cool $\left(T_{\text {eff }}=6930 \mathrm{~K}\right)$, weakly magnetic star is over abundant in Nd by a factor of about 275 relative to the standard abundance distribution (SAD, Grevesse \& Sauval 1996). Her calculations give $\log \left(N_{\mathrm{Nd}} / N_{\text {Total }}\right)=-8.0 \pm 0.3$ from Nd III assuming $\xi_{\mathrm{t}}=0.85 \mathrm{~km} \mathrm{~s}^{-1}$.
In summary, we have presented $g f$-values and Landé $g$-factors for the RE ion Nd III for a number of lines in the optical region with accuracies sufficient to facilitate abundance work (and certain other types of stellar atmosphere calculations) in CP stars. These results have been applied in the analysis of HD 101065 and yield abundances with uncertainties consistent with the errors in the $\log (g f)$ values, plus an additional $0.1-0.2$ dex attributable to the choice of parameters used in constructing the stellar model atmosphere and in treating line broadening (e.g., rotation, instrumental and Zeeman effects, hfs, etc.) in this very unusual star.

The $g f$-values reported here are not generally comparable in precision to the best ones available from highly sophisticated multi-configuration Dirac-Fock approaches (cf. Brage et al. 1999), or from enhanced Cowan-code calculations conditioned by new, extensive energy level data (cf. Palmeri et al. 2000 on Pr III), where uncertainties as low as $15 \%(\approx \pm 0.06 \mathrm{dex})$ are attainable. The former type of calculation, because of its complexity and the number of active orbitals required for ions like Nd III, is not likely to be carried out except perhaps for a small number of lines of special importance. The latter method, which depends on a significant expansion of the energy level structure, requires an extended analysis of the spectrum (which for Nd III is now underway [Wyart 2000]) for its successful implementation. For global abundance studies, data of the type and quality given here will thus remain useful until additional laboratory measures are made and/or more extensive theoretical work is performed. We encourage both avenues of research to be pursued.

Acknowledgements. The author expresses his appreciation to Dr. Tanya Ryabchikova for her continuing encouragement to make these data available to broader community of workers in this field, and for her generous provision of the results of her studies of the abundances of $\mathrm{NdIII}$ in roAp stars in advance of their publication. Additional thanks are due to Dr. Charles R. Cowley for his assistance with the REE abundance analysis of HD 101065. The author also acknowledges helpful correspondance with Dr. E. Biémont, Dr. J.-F. Wyart, and Dr. R.D. Cowan during the preparation of this manuscript for publication.

\section{References}

Aikman G.C.L., Cowley C.R., Crosswhite H., 1979, ApJ 232, 812

Baschek B., 1995, Summary and Outlook, Future Needs for Atomic Data, in: Astrophysical Applications of Powerful New Databases, Adelman S.J., Wiese W.L. (eds.), ASP Conf. Ser. 78, 527

Bidelman W.P., Cowley C.R., Iler A.L., 1995, Publ. Obs. U. Mich. 12, 77

Bord D.J., Barisciano Jr. L.P., Cowley C.R., 1996, MNRAS 278, 997

Bord D.J., Cowley C.R., Norquist P.L., 1997, MNRAS 284, 869 
Bord D.J., Cowley C.R., Mirijanian D., 1998, Solar Phys. 178, 221

Brage T., Leckrone D.S., Proffitt C.R., 1999, ApJ 513, 524

Cowan R.D., 1981, The Theory of Atomic Structure and Spectra. Berkeley: Univ. California Press

Cowan R.D., 1985, Programs RCN/RCN2/RCG/RCE, Los Alamos Natl. Lab.

Cowley C.R., 1976, ApJS 32, 631

Cowley C.R., 1996, Automatic Spectrum Synthesis, in: Model Atmospheres and Spectrum Synthesis, Adelman S.J., Kupka F., Wiese W. (eds.), ASP Conf. Ser. 108, 170

Cowley C.R., 1999 (private communication)

Cowley C.R., Bord D.J., 1998, The Third Spectrum of Neodymium: Applications to HR 6870 and $\gamma$ Equulei, in: The Scientific Impact of the Goddard High Resolution Spectrograph, Brandt J.C., Ake III T.B., Petersen C.C. (eds.), ASP Conf. Ser. 143, 346

Cowley C.R., Crosswhite H.M., 1978, PASP 90, 108

Cowley C.R., Mathys G., 1998, A\&A 396, 666

Cowley C.R., Bidelman W.P., Mathys G., 1998, Bull. AAS 30, 1317

Cowley C.R., Ryabchikova T.A., Kupka F., Bord D.J., Mathys G., Bidelman W.P., 2000, MNRAS (accepted)

Crosswhite H., 1976 (private communication)

Gaigalas G., 1998 (private communication)

Goldschmidt Z.B., 1978, Atomic Properties (Free Atoms), in: Handbook on the Physics and Chemistry of Rare Earths, Vol. 1, Gschneidner Jr. K.A., Eyring L. (eds.). Amsterdam: North-Holland Publ. Co., p. 1

Gratton R.G., Sneden C., 1994, A\&A 287, 927

Grevesse N., Sauval A.J., 1996, Standard Abundances, in: Cosmic Abundances, Holt S., Sonnenborn G. (eds.), ASP Conf Ser. 99, 117

Harrison G.R., Rosen N., McNally Jr. J.R., 1945, JOSA 35, 658

Judd B.R., 1962, Phys. Rev. 125, 613

Kupka F., Piskunov N.E., Ryabchikova T.A., Stempels H.C., Weiss W.W., 1999, A\&AS 138, 119

Leckrone D.S., Johansson S.G., Wahlgren G.M., 1998, GHRS Spectroscopy of CP Stars: The $\chi$ Lupi Pathfinder Project, in: The Scientific Impact of the Goddard High Resolution Spectrograph, Brandt J.C., Ake III T.B., Petersen C.C. (eds.), ASP Conf. Ser. 143, 135

Martin W.C., Zalubas R., Hagan L., 1978, Atomic Energy Levels - The Rare Earth Elements, NSRDS-NBS 60.
Washington: U.S. Gov. Print. Off. (MZH)

Meggers W.F., Corliss C.H., Scribner B.F., 1975, Tables of Spectral Line Intensities, NBS Mono. 145. Washington: U.S. Gov. Print. Off.

Palmeri P., Quinet P., Frémat Y., Wyart J.-F., Biémont E., 2000, ApJS (in press)

Piskunov N.E., Kupka F., Ryabchikova T.A., Wiese W.L., Jeffrey C.S., 1995, A\&AS 112, 525

Proffitt C.R., Brage T., Leckrone D.S., et al., 1999, ApJ 512, 942

Quinet P., Palmeri P., Biémont E., et al., 1999, MNRAS 307, 934

Ryabchikova T.A., Davidova E.S., Adelman S.J., 1990, PASP 102,581

Ryabchikova T., Piskunov N., Savanov I., Kupka F., Malanushenko V., 1999, A\&A 343, 229

Ryabchikova T.A., Savanov I.S., Malanushenko V.P., Kudryavtsev D.O., 1999a, Astron. Reports (submitted)

Ryabchikova T.A., Savanov I.S., Hatzes A.P., Weiss W.W., Handler G., 2000 (in preparation)

Sugar J., Reader J., 1973, J. Chem. Phys. 59, 2083

Vaeck N., Godefroid M., Froese Fischer C., 1992, Phys. Rev. A 46,3704

Wiese W.L., Fuhr J.R., Kelleher D.E., Martin W.C., Musgrove A., Sugar J., 1995, Critically Evaluated Data for Atomic Spectra, in: Astrophysical Applications of Powerful New Databases, Adelman S.J., Wiese W.L. (eds.), ASP Conf. Ser. 78, 105

Wolff S.C., Hagen W., 1976, PASP 88, 119

Wyart J.-F., 2000 (private communication)

Wyart J.-F., Palmeri P., 1998, Phys. Scr. 58, 368

\section{List of objects}

"HD 101065" on page 517

"HD 122970" on page 517

" $\gamma$ Equulei" on page 517

"HR 6870" on page 517

"HR 465 " on page 518

"HD 51418" on page 521

"HD 200311" on page 521

"HD 192913" on page 521 